\title{
A Domain Decomposition Method for Hybrid Shell Vector Element with Boundary Integral Method
}

\author{
Lin Lei, Jun Hu, and Hao-Quan Hu \\ School of Electronic Engineering, University of Electronic Science and Technology of China, Chengdu 611731, China
}

Correspondence should be addressed to Jun Hu, hujun@uestc.edu.cn

Received 8 June 2012; Accepted 1 August 2012

Academic Editor: Zhongxiang Q. Shen

Copyright () 2012 Lin Lei et al. This is an open access article distributed under the Creative Commons Attribution License, which permits unrestricted use, distribution, and reproduction in any medium, provided the original work is properly cited.

For the conducting body coated with thin-layer material, plenty of fine meshes are required in general. In this paper, shell vector element (SVE) is used for modeling of thin coating dielectric. Further, a domain decomposition (DD) method for hybrid shell vector element method boundary integral (SVE-BI) is proposed for analysis of electromagnetic problem of multiple threedimensional thin-coating objects. By this method, the whole computational domains are divided into sub-SVE domains and boundary element domains. With shell element, not only the unknowns are far less than the one by traditional vector element method, but only surface integral is required. The DDM framework used for hybrid SVE-BI also enhances the computational efficiency of solving scattering from multiple coating objects greatly. Finally, several numerical examples are presented to prove the accuracy and efficiency of this DDM-SVE-BI method.

\section{Introduction}

Recently electromagnetic scattering from multiple conducting bodies coated by thin-layer dielectric has attracted more and more interest. Typical applications can be found in scattering from composite conductor and dielectric, microwave integrated circuits design, analysis of antenna array, and so on.

For conductor structures coated by thin-layer material, many numerical methods have been developed. Integral equation methods based on thin dielectric sheet approximation (TDS) [1-5] avoid the volumetric discretization of material region, the computational region is only limited as the surface of conductor. A multilevel-TDS extension is also proposed for solution of conducting body coated by multithin-layer materials $[6,7]$. For lossy thin-layer coating, the impedance boundary condition (IBC) is also developed to simplify the electromagnetic analysis by building the relation between the equivalent magnetic current and electric current on the surface of conductor [8]. A rigorous moment method solution of composite bodies with thin coating dielectric is also shown in [9].

As well known, finite element method (FEM) is also widely used for analysis of composite conducting body and dielectric because of its powerful ability of modeling inhomogeneous materials. In order to combine together the advantage of FEM and integral equation, the hybrid FEM with boundary integral method (FEM-BI) is proposed [1012]. Though FEM-BI has a good computational property for composite structures, it is deficient for the analysis of conductor structures coated by thin-layer material. This is because plenty of fine meshes will be required for modeling thin layers if using traditional elements like tetrahedral elements. To further reduce the total number of unknowns required in FEM, shell vector elements (SVE) [13] are developed, respectively. The SVEs are also extended into FEM-BI framework by us [14]. A remarkable advantage of using SVEs is that the volume integral can be simplified into surface integral.

To realize efficient analysis of complex structures, domain decomposition method (DDM) is developed based on FEM-BI framework $[15,16]$. The FEM is chosen for interior regions of subdomains and the $\mathrm{BI}$ is chosen for the rest of the system. In this paper, a novel DDM is developed based on hybrid SVE and BI method (SVE-BI) in order to realize fast solution of multiple conducting bodies coated by thin-layer dielectric. By this method, the original problem is divided into many internal domains and external domains. 


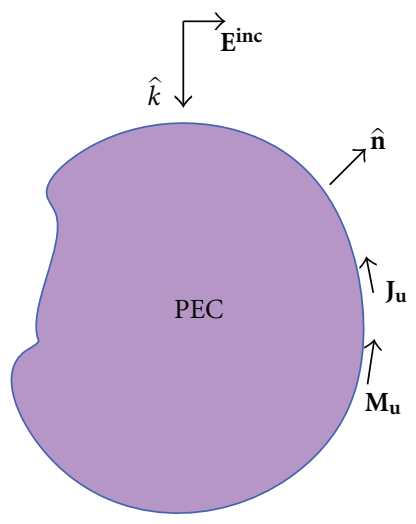

Figure 1: The 3D electromagnetic scattering problem of conducting object coated by thin dielectric.

For internal domains, SVE is used to reduce the number of unknowns. For external domains, BI method is adopted to take into account all interactions between equivalent electric current and magnetic current on the boundary of each object.

The rest of the paper is organized as follows: the hybrid SVE-BI method is briefly introduced in Section 2. In Section 3, we demonstrate the DDM-SVE-BI method. Finally, several numerical examples are presented to demonstrate the efficiency and accuracy of this method. The conclusions are also given.

\section{Hybrid Shell Vector Element and Boundary Integral Method (SVE-BI)}

For sake of simplicity, 3D electromagnetic scattering problem of conducting object coated by thin dielectric illuminated by an incident wave $\mathbf{E}^{\text {inc }}$ is considered, as shown in Figure 1. The $\hat{\mathbf{n}}$ is the unit normal vector on surface of the object. In the SVE-BI method, the SVE is used in dielectric domain of the object, the BI is used on the surface of the object. Here, $\mathbf{J}_{\mathbf{u}}$, $\mathbf{M}_{\mathbf{u}}$ are the equivalent surface electric and magnetic currents on the surface.

The E field inside dielectric domain of object satisfies the following equation:

$$
\nabla \times\left(\boldsymbol{\mu}_{\mathbf{r}}^{-1} \nabla \times \mathbf{E}\right)-k_{0}^{2} \boldsymbol{\varepsilon}_{\mathbf{r}} \mathbf{E}=0
$$

where $\boldsymbol{\mu}_{\mathrm{r}}, \boldsymbol{\varepsilon}_{\mathrm{r}}$ denotes the relative permeability and permittivity of the dielectric, respectively. $k_{0}$ is the wave number in free space.

The boundary conditions on the surface of object are written as:

$$
\begin{gathered}
\hat{n} \times\left.\mathbf{E}\right|_{S^{-}}=\hat{n} \times\left.\mathbf{E}\right|_{S^{+}} \\
\hat{n} \times\left.\left(\frac{1}{\boldsymbol{\mu}_{\mathbf{r}}} \nabla \times \mathbf{E}\right)\right|_{S^{-}}=-j k_{0} \hat{n} \times\left.\overline{\mathbf{H}}\right|_{S^{+}},
\end{gathered}
$$

where $\overline{\mathbf{H}}=\eta_{0} \mathbf{H}, \eta_{0}$ is the wave impedance in free space.
The functional $F(\mathbf{E})$ is expressed as follow:

$$
\begin{gathered}
F(\mathbf{E})=\frac{1}{2} \iiint_{V}\left[\boldsymbol{\mu}_{\mathbf{r}}^{-1}(\nabla \times \mathbf{E}) \cdot(\nabla \times \mathbf{E})\right. \\
\left.-k_{0}^{2} \boldsymbol{\varepsilon}_{\mathbf{r}} \mathbf{E} \cdot \mathbf{E}\right] d V \\
+j k_{0} \eta_{0} \iint_{S} \hat{n} \cdot\left(\mathbf{E} \times \mathbf{H}_{\mathbf{u}}\right) d S .
\end{gathered}
$$

Finally, the FEM matrix equation of the object is yielded from (3):

$$
[K]\{E\}+[B]\left\{H_{u}\right\}=\{0\},
$$

where $E$ is the electric field expansion coefficient inside the object. $H_{u}$ is the magnetic field expansion coefficient on the object surface. The matrix $K$ comes from the volume integration, matrix $B$ comes from the surface integration.

In the SVE-BI method, integral equation method is applied on the surface of the object. As shown in [14], the matrix is written as follows:

$$
[P]\left\{E_{u}\right\}+[Q]\left\{H_{u}\right\}=\{b\},
$$

where $P$ and $Q$ come from the surface integration.

Combing (4) and (5), all unknown coefficients can be solved.

For the surface integral term in (3), the magnetic field on the surface can be expanded by three edges of planar triangle on surface $\mathbf{S}$ as follows:

$$
\mathbf{H}_{\mathbf{u}}=\sum_{j=1}^{3} H_{j}^{s} \mathbf{N}_{\mathbf{j}}^{\mathbf{s}},
$$

where $H_{j}^{s}, \mathbf{N}_{\mathbf{j}}^{\mathbf{s}}$ are the unknown coefficient and the basis function of the $j$ th edge, respectively.

By the SVE, the electric field is expanded as follows:

$$
\mathbf{E}^{\mathbf{e}}=\sum_{j=1}^{3}\left(E_{j}^{e} \beta \mathbf{N}_{\mathbf{j}}+E_{j}^{\prime e} \beta^{\prime} \mathbf{N}_{\mathbf{j}}^{\prime}\right)+\sum_{j=1}^{3} E_{n j}^{e} L_{j}^{e} \widehat{\mathbf{n}},
$$

where $\beta^{\prime}=1-\beta, \mathbf{N}_{\mathbf{j}}=\left(L_{j 1}^{e} \nabla L_{j 2}^{e}-L_{j 2}^{e} \nabla L_{j 1}^{e}\right) \mathbf{l}_{j}^{e}, \mathbf{N}_{\mathbf{j}}^{\prime}=$ $\left(L_{j 1}^{e} \nabla L_{j 2}^{e}-L_{j 2}^{e} \nabla L_{j 1}^{e}\right) l_{j}^{e}, E_{j}^{e}$ : the expansion coefficient of the $j$ th edge vector in upper triangle, $E_{j}^{\prime}$ : the expansion coefficient of the $j$ th edge vector in bottom triangle, and $E_{n j}^{e}$ : the expansion coefficient of normal vector at the node- $j$.

The shell element is the degenerated prism element. As shown in Figure 2, there are total six edge vectors along the corresponding edges in the upper triangle and bottom triangle, and three normal vectors.

A linear function $\beta(\varsigma)$ was used to describe the variation of the field along the normal direction, and $\nabla \beta=-\hat{\mathbf{n}} / \mathbf{d}$. The $\beta \mathbf{N}_{\mathbf{j}}(j=1,2,3), \beta^{\prime} \mathbf{N}_{\mathbf{j}}^{\prime}(j=1,2,3)$ are the edge basis functions in the upper and bottom triangle, respectively. The $L_{j}, j=1,2,3$ is the normal basis function at the node- $j$.

For the SVE, as shown in [14], the integration along the normal direction can be calculated analytically. So the volume integral can be simplified into surface integral. More details about the SVE-BI can be found in [14].

For conducting objects coated by thin-layer material, the electric field in the bottom surface of shell vector element must be zero, only the integral in the upper surface is needed. 


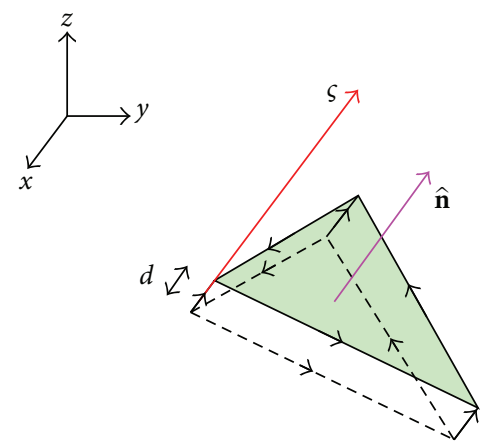

(a) the prism vector element

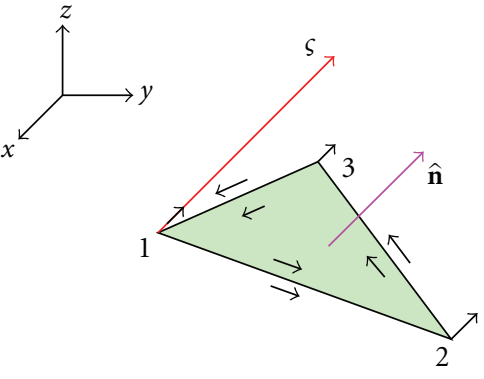

(b) the shell vector element

FIGURE 2: The structure of the prism vector element and the shell vector element.

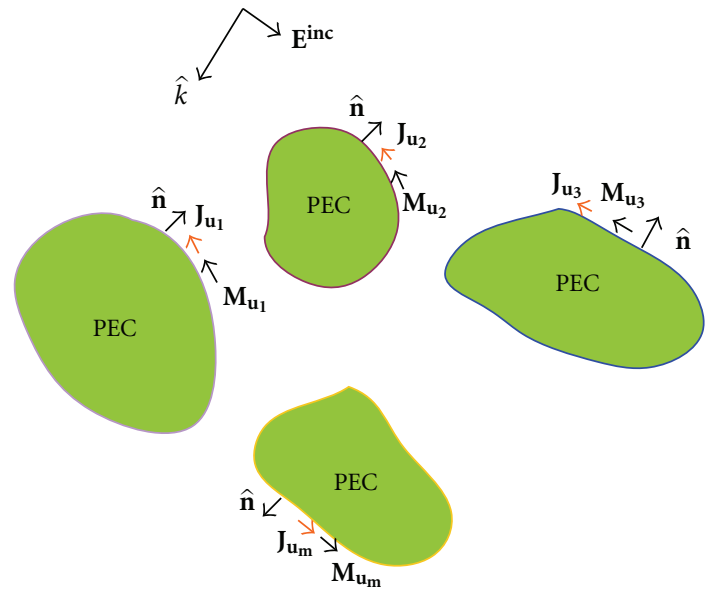

FIgURE 3: The 3D electromagnetic scattering problem of multiple conducting bodies coated with thin layer dielectric.

\section{The DDM-SVE-BI Method}

For multiple conducting bodies coated with thin dielectric as shown in Figure 3, the whole computational domains are divided into a lot of sub-SVE domains and boundary element domains. For each sub-SVE domain, namely, volume domain of each object, equation (4) can be expressed as follows:

$$
\left[\begin{array}{l}
\mathbf{K}_{\mathrm{nn}} \mathbf{K}_{\mathrm{nu}} \\
\mathbf{K}_{\mathrm{un}} \mathbf{K}_{\mathrm{uu}}
\end{array}\right]_{\mathrm{m}}\left\{\begin{array}{l}
\mathbf{E}_{\mathbf{n}_{\mathrm{m}}} \\
\mathbf{E}_{\mathbf{u}_{\mathrm{m}}}
\end{array}\right\}=-\left[\begin{array}{c}
\mathbf{0} \\
\mathbf{B}
\end{array}\right]_{\mathrm{m}}\left\{\mathbf{H}_{\mathbf{u}_{\mathrm{m}}}\right\},
$$

where $\mathbf{E}_{\mathbf{n}_{\mathrm{m}}}$ is the normal electric field expansion coefficient at each node of the SVEs of the $m$ th object, which is a column matrix of $\mathbf{N}_{\mathbf{n}_{m}} \times \mathbf{1}$. $\mathbf{E}_{\mathbf{u}_{\mathrm{m}}}$ is the electric field expansion coefficient along each edge of the SVEs of the $m$ th object, which is a column matrix of $\mathbf{N}_{\mathbf{u}_{\mathrm{m}}} \times \mathbf{1}$. In (8), $\mathbf{K}_{\mathbf{n n}}$ is a square matrix of $\mathbf{N}_{\mathbf{n}_{\mathrm{m}}} \times \mathbf{N}_{\mathbf{n}_{\mathrm{m}}}, \mathbf{K}_{\mathbf{n u}}$ is a matrix of $\mathbf{N}_{\mathbf{n}_{\mathrm{m}}} \times \mathbf{N}_{\mathbf{u}_{\mathrm{m}}}, \mathbf{K}_{\mathbf{u n}}$ is a matrix of $\mathbf{N}_{\mathbf{u}_{m}} \times \mathbf{N}_{\mathbf{n}_{m}}, \mathbf{K}_{\mathbf{u u}}$ is a square matrix of $\mathbf{N}_{\mathbf{u}_{m}} \times \mathbf{N}_{\mathbf{u}_{m}}$, B is a square matrix of $\mathbf{N}_{\mathbf{u}_{m}} \times \mathbf{N}_{\mathbf{u}_{m}}, \mathbf{H}_{\mathbf{u}_{\mathrm{m}}}$ is a column matrix of $\mathbf{N}_{\mathbf{u}_{m}} \times \mathbf{1} . \mathbf{N}_{\mathbf{n}_{\mathrm{m}}}, \mathbf{N}_{\mathbf{u}_{\mathrm{m}}}$ is the number of nodes, the number of edges of all SVEs of the $m$ th object, respectively.
Equation (8) can be written as:

$$
\left\{\begin{array}{l}
E_{\mathbf{n}_{\mathrm{m}}} \\
\mathbf{E}_{\mathbf{u}_{\mathrm{m}}}
\end{array}\right\}=-\left[\begin{array}{l}
\mathbf{K}_{\mathbf{n n}} K_{\mathbf{n u}} \\
\mathbf{K}_{\mathbf{u n}} \mathbf{K}_{\mathbf{u u}}
\end{array}\right]_{m}^{-1}\left[\begin{array}{l}
\mathbf{0} \\
\mathbf{B}
\end{array}\right]_{\mathrm{m}}\left\{\mathbf{H}_{\mathbf{u}_{\mathrm{m}}}\right\} .
$$

Extracting surface electric field expansion coefficient $\mathbf{E}_{\mathbf{u}_{\mathrm{m}}}$ from (9):

$$
\left\{\mathbf{E}_{\mathbf{u}_{\mathrm{m}}}\right\}=\left[\mathbf{X}_{\mathbf{C}_{\mathbf{m}}}\right]\left\{\mathbf{H}_{\mathbf{u}_{\mathbf{m}}}\right\},
$$

where $\mathbf{X}_{\mathbf{C}_{\mathrm{m}}}$ is a square matrix of $\mathbf{N}_{\mathbf{u}_{\mathrm{m}}} \times \mathbf{N}_{\mathbf{u}_{\mathrm{m}}}$. According to the present DDM, the computation of $\mathbf{X}_{\mathrm{C}_{\mathrm{m}}}$ can be easily parallelized, the computational time can be saved greatly. Because of the sparse property of matrix $[\mathbf{K}]$, the iterative method usually is used to attain $\mathbf{X}_{\mathbf{C}_{\mathrm{m}}}$. But it is not always successful, and it costs too much time when the condition number of matrix $[\mathbf{K}]$ is not very well. In this paper, the inverse of matrix is used for computation of $\mathbf{X}_{\mathbf{C}_{m}}$ to keep good accuracy. Here, the direct Gaussian Elimination is used for attaining $\mathbf{X}_{\mathrm{C}_{\mathrm{m}}}$.

Substitute (10) into (5), we obtain,

$$
\left([\mathbf{P}]\left[\begin{array}{cccc}
\mathbf{X}_{\mathbf{C}_{1}} & & & \\
& \mathbf{X}_{\mathbf{C}_{2}} & & \\
& & \cdots & \\
& & & \mathbf{X}_{\mathbf{C}_{\mathrm{m}}}
\end{array}\right]+[\mathbf{Q}]\right)\left[\mathbf{H}_{\mathbf{u}}\right]=[\mathbf{b}] .
$$

Here, $[\mathbf{P}]$ and $[\mathbf{Q}]$ is a square matrix of $\mathbf{N}_{\mathbf{u}} \times \mathbf{N}_{\mathbf{u}}, \mathbf{H}_{\mathbf{u}}$ is a column matrix of $\mathbf{N}_{\mathbf{u}} \times \mathbf{1}, \mathbf{N}_{\mathbf{u}}=\mathbf{N}_{\mathbf{u}_{1}}+\mathbf{N}_{\mathbf{u}_{2}}+\cdots \mathbf{N}_{\mathbf{u}_{\mathrm{m}}}$ denotes the summation of surface unknowns along the edges about all the objects. For solving (11), direct Gaussian Elimination technique is used to attain accurate results.

\section{Numerical Results}

To demonstrate the accuracy and efficiency of the present method, some typical numerical results are shown here.

4.1. Two Dielectric Sphere. The first example is two separated dielectric spheres, shown in Figure 4. The frequency is $300 \mathrm{MHz}$. The radius of the two dielectric spheres are $0.2 \mathrm{~m}$. The distance between the centers of two spheres is $0.8 \mathrm{~m}$. The relative permittivity is $\varepsilon_{r}=2.0$. The incident angle of 


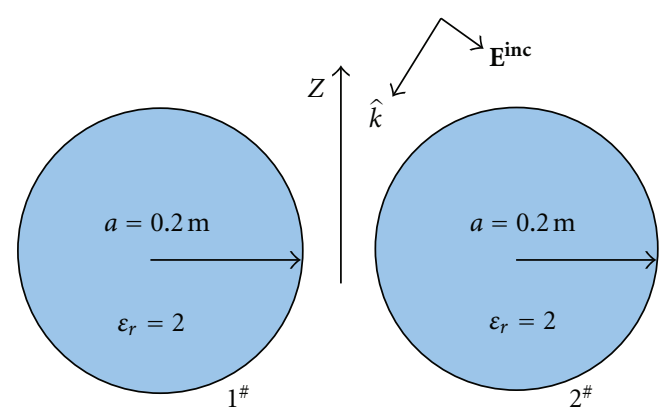

Figure 4: Two dielectric spheres.

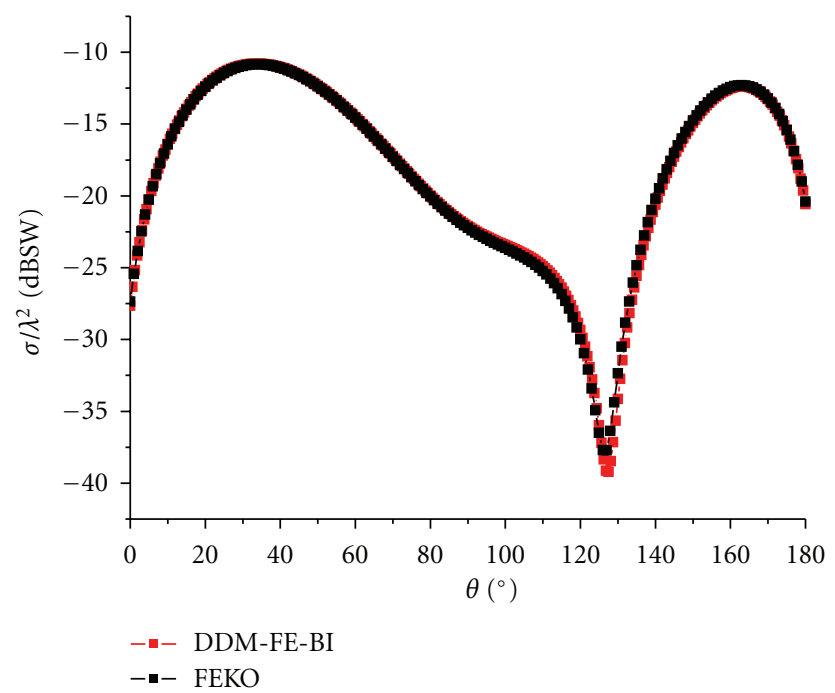

FIgure 5: The Bistatic RCS of two dielectric spheres.

plane wave is $\theta=45^{\circ}, \varphi=0^{\circ}$ the bistatic RCS in horizontal polarization is computed. Here, DDM for FEM-BI is used to calculate scattering from two dielectric spheres in order to prove the accuracy of DDM. From Figure 5, it is shown clearly that the results by DDM-FE-BI agree well with the one by commercial software FEKO based on the surface integral equation with PMCHWT formulation [17].

4.2. $3 \times 2$ Array of Coating PEC Spheres. The second example is to solve scattering of $3 \times 2$ array of coating PEC spheres located in the $x-y$ plane, as shown in Figure 6 . The radius of PEC sphere is $0.2 \mathrm{~m}$. The thickness of dielectric coating is $0.05 \mathrm{~m}$ with $\varepsilon_{r}=2.0, \mu_{r}=1.0$. The distance between the centers of two elements is $0.8 \lambda_{0}$ in both the $x$ - and $y$ dimension as shown in Figure 6. The incident electric field is $\hat{x}$ polarized plane wave propagating into the negative $\hat{z}$ direction at $0.3 \mathrm{GHz}$. The results by Moment of Method $(\mathrm{MoM})$ are also given for comparison.

As shown in Figure 7, the results by DDM-SVE-BI agree with the one by DDM-FE-BI and the result of the MoM very well.

The comparison of the DDM-FE-BI and DDM-SVEBI is shown in Table 1. Table 1 demonstrates that the unknowns, the memory requirement of matrix $\mathbf{K}$ for single

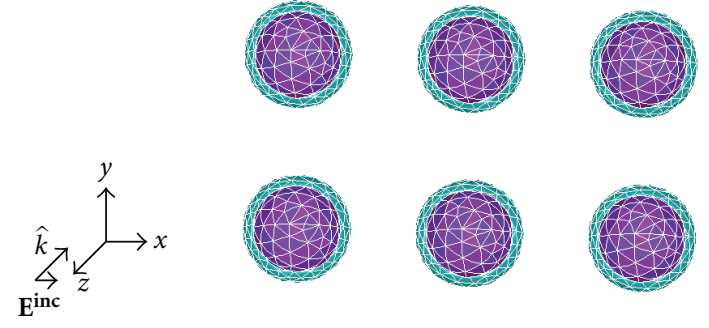

Figure 6: $3 \times 2$ array of coating PEC spheres.

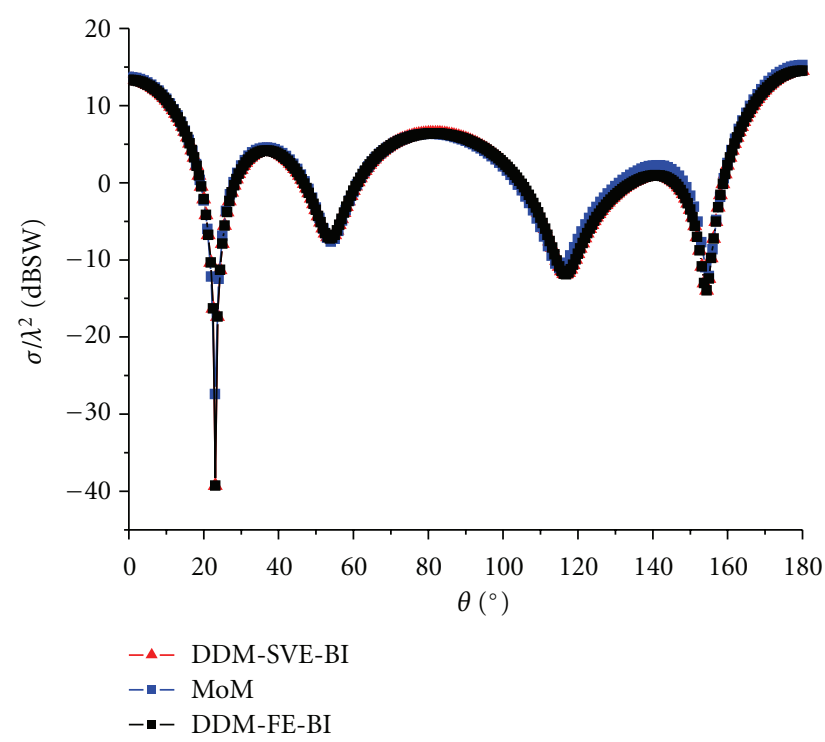

Figure 7: The Bistatic RCS of $3 \times 2$ array of coating PEC spheres.

TABLE 1: The comparison of the DDM-FE-BI and DDM-SVE-BI. Bistatic RCS of $3 \times 2$ array of coating PEC spheres. (mesh density with $\left.\lambda_{0} / 10\right)$.

\begin{tabular}{lcc}
\hline Method & DDM-FE-BI & DDM-SVE-BI \\
\hline $\begin{array}{l}\text { Unknowns of single PEC } \\
\text { coating sphere }\end{array}$ & 523 & 274 \\
$\begin{array}{l}\text { The total unknowns } \\
\begin{array}{l}\text { Memory of matrix } \boldsymbol{K} \text { for } \\
\text { single coating PEC sphere }\end{array}\end{array}$ & 3138 & 1644 \\
$\begin{array}{l}\text { Computational time for } \\
\text { matrix } \mathbf{X}_{\mathbf{C}}\end{array}$ & $2.2 \mathrm{Mb}$ & $0.6 \mathrm{Mb}$ \\
\hline
\end{tabular}

coating PEC sphere with the SVEs is only $52 \%, 27 \%$ of the traditional tetrahedral elements, respectively. The CPU time for attaining single $\mathbf{X}_{\mathbf{C}}$ by the SVEs is only $20 \%$ of the one by traditional tetrahedral elements.

Obviously, the advantages of the DDM-SVE-BI over the DDM-FE-BI on reducing the number of unknowns and computational time are very remarkable.

To further prove the accuracy of the present method, the RCS of this same $3 \times 2$ array with magnetic material coating is also computed, the material parameters are $\varepsilon_{r}=$ $1.0, \mu_{r}=2.0-j 1.5$. The same incident angle with $\hat{x}$ polarized 


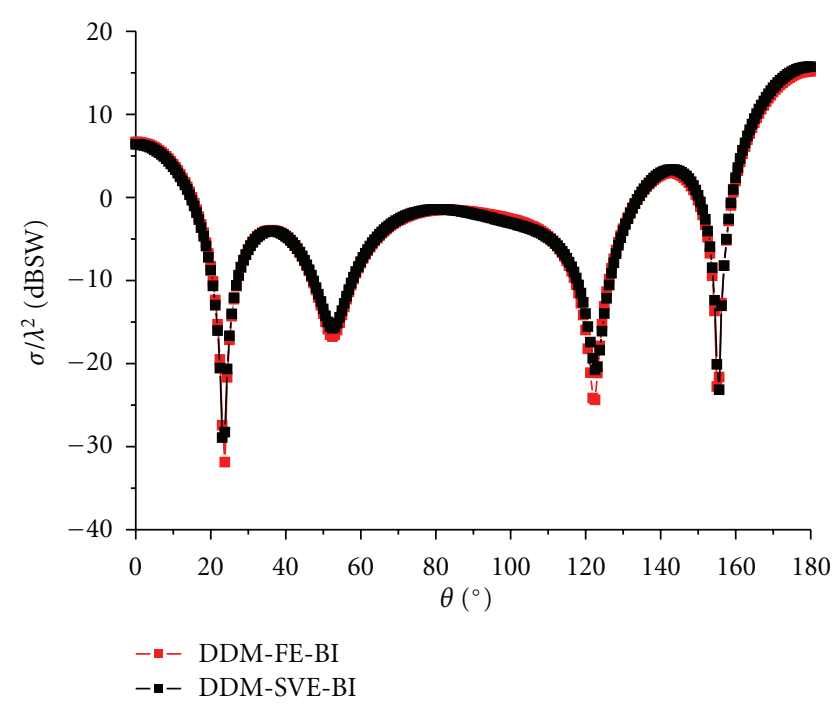

Figure 8: The Bistatic RCS of $3 \times 2$ array of coating PEC spheres with magnetic material.

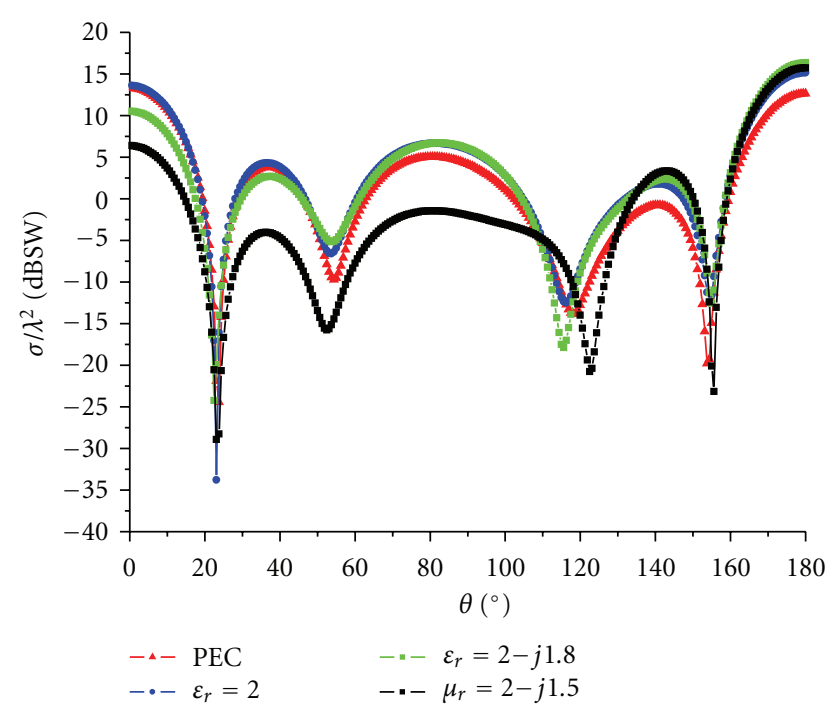

Figure 9: The Bistatic RCS of $3 \times 2$ array of coating PEC spheres with different materials.

is analyzed. As shown in Figure 8, the results of DDM-SVE$\mathrm{BI}$ also agree with the results of DDM-FE-BI very well.

To further investigate the influence of different coating materials on the RCS of this $3 \times 2$ array, four different coating materials with $\varepsilon_{r}=2.0, \mu_{r}=1.0, \varepsilon_{r}=2.0-j 1.8, \mu_{r}=1.0$, $\varepsilon_{r}=1.0, \mu_{r}=2.0-j 1.5$ including PEC are evaluated. As shown in Figure 9, compared with the RCS from $3 \times 2$ PEC array, two lossy coating materials reduce the backscattering RCS greatly. The RCS reduction achieves $2.8 \mathrm{~dB}, 6.9 \mathrm{~dB}$ for the case of $\varepsilon_{r}=2.0-j 1.8, \mu_{r}=1.0, \varepsilon_{r}=1.0, \mu_{r}=2.0-j 1.5$, respectively. For this structure, lossless coating material with $\varepsilon_{r}=2.0, \mu_{r}=1.0$ has little influence on the backscattering RCS. For the forward RCS, it is enhanced about $3 \mathrm{dBSW}$ in all four cases. It is also demonstrated from Figure 9 that the

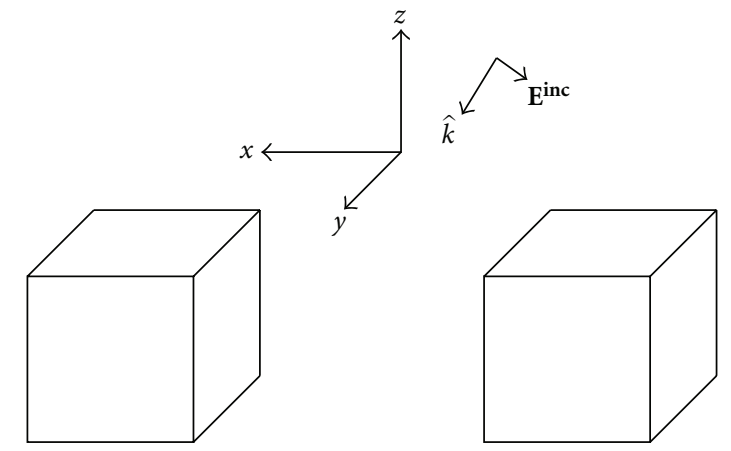

Figure 10: Two coating PEC cubes.

TABLE 2: The comparison of the DDM-FEM-BI and DDM-SVE-BI. Bistatic RCS of two coating PEC cubes. (mesh density with $\lambda_{0} / 20$ ).

\begin{tabular}{lcc}
\hline Method & DDM-FE-BI & DDM-SVE-BI \\
\hline $\begin{array}{l}\text { Unknowns of single PEC } \\
\text { coating cube }\end{array}$ & 5344 & 3218 \\
$\begin{array}{l}\text { The total unknowns } \\
\begin{array}{l}\text { Memory of matrix K about } \\
\text { single PEC coating cube }\end{array}\end{array}$ & 10688 & 6436 \\
$\begin{array}{l}\text { Computational time for } \\
\text { matrix } \mathbf{X}_{\mathrm{C}}\end{array}$ & $228.5 \mathrm{Mb}$ & $82.8 \mathrm{Mb}$ \\
\hline
\end{tabular}

magnetic lossy coating material has great influence on the RCS of this array.

4.3. Two Coating PEC Cubes. The third example is two coating PEC cubes, shown in Figure 10. The frequency is $300 \mathrm{MHz}$. The length of the cube is $0.5 \mathrm{~m}$, the thickness of the coated layer is $0.02 \mathrm{~m}$. The distance between the centers of two cubes is $1.6 \mathrm{~m}$. The relative permittivity $\varepsilon_{r}=2.0$. The incident angle of plane wave is $\theta=45^{\circ}, \varphi=0^{\circ}$, the bistatic RCS in horizontal polarization is computed. From Figure 11, it is shown clearly that the results by the DDM-SVE-BI agree well with the one by the DDM-FEM-BI.

The comparison of the DDM-SVE-BI over the DDM-FE$\mathrm{BI}$ is shown in Table 2. The unknowns, the memory of matrix $\mathbf{K}$ for single coating PEC cube meshed with the SVEs is only $60 \%, 36 \%$ of the one with traditional tetrahedral elements, respectively. The CPU time for attaining single $\mathbf{X}_{\mathbf{C}}$ by the SVEs is only $25.8 \%$ of the one by tetrahedral elements.

Obviously, compared with the DDM-FE-BI, the DDMSVE-BI can save the memory and CPU time greatly for multiple thin-coating objects.

\section{Conclusions}

In this paper, the SVE-BI based on the DDM framework (DDM-SVE-BI) is proposed for scattering analysis of multiple conducting bodies coated with thin layer dielectric. The whole computational domains are divided into subSVE domains and boundary element domains. Compared with traditional vector element method, the DDM-SVE-BI 


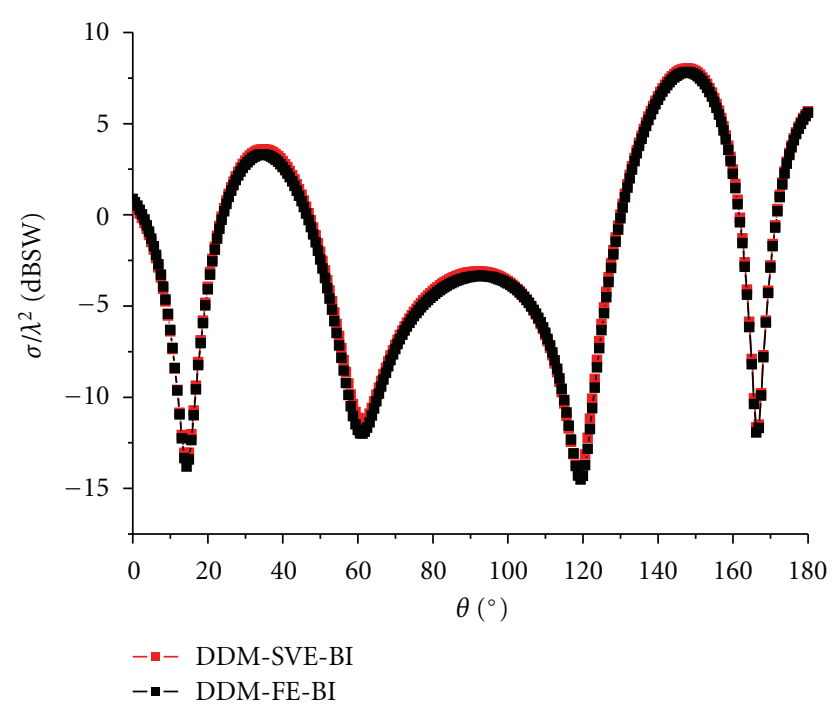

Figure 11: The Bistatic RCS of two coating PEC cubes.

reduces the unknowns greatly, enhances the computational efficiency of solving scattering from multiple coating objects.

Because linear basis function is used to represent the normal component of electric field in coating materials, this method based on shell vector basis is very efficient for solving thin coating problems, even multiple thin coating problems. It is valid for thin coating materials with thickness of up to 0.1 dielectric wavelength. On the other hand, this method is limited to solve the objects at resonant region. To solve large scale problems, fast methods are necessary to implement into it. This will be our next work.

\section{Acknowledgment}

The work is supported by the Nature Science Foundation of China (no. 60971032).

\section{References}

[1] R. F. Harrington and J. R. Mautz, "An impedance sheet approximation for thin dielectric shells," IEEE Transactions on Antennas and Propagation, vol. 23, no. 4, pp. 531-534, 1975.

[2] C. Lu, "A modified thin dielectric approximation for calculation of em scattering by dielectric objects with thin material coating," in Proceedings of the IEEE Antennas and Propagation Society International Symposium, pp. 2809-2812, June 2007.

[3] I. T. Chiang and W. C. Chew, "Thin dielectric sheet simulation by surface integral equation using modified RWG and pulse bases," IEEE Transactions on Antennas and Propagation, vol. 54, no. 7, pp. 1927-1934, 2006.

[4] I. T. Chiang and W. C. Chew, "A coupled PEC-TDS surface integral equation approach for electromagnetic scattering and radiation from composite metallic and thin dielectric objects," IEEE Transactions on Antennas and Propagation, vol. 54, no. 11, pp. 3511-3516, 2006.

[5] G. Pan and R. M. Narayanan, "Electromagnetic scattering from a dielectric sheet using the method of moments with approximate boundary condition," Electromagnetics, vol. 24, no. 5, pp. 369-384, 2004.
[6] S. He, Z. Nie, J. Wei, and J. Hu, "Numerical solution for dielectric-coated PEC targets based on multi-layer TDS approximation," in Proceedings of the Asia Pacific Microwave Conference (APMC '08), December 2008.

[7] S. He, Z. Nie, J. Wei, and J. Hu, "A highly efficient numerical solution for dielectric-coated PEC targets," Waves in Random and Complex Media, vol. 19, no. 1, pp. 65-79, 2009.

[8] D. S. Wang, "Limits and validity of the impedance boundary condition on penetrable surfaces," IEEE Transactions on Antennas and Propagation, vol. 35, no. 4, pp. 453-457, 1987.

[9] P. M. Goggans, A. A. Kishk, and A. W. Glisson, "A systematic treatment of conducting and dielectric bodies with arbitrarily thick or thin features using the method of moments," IEEE Transactions on Antennas and Propagation, vol. 40, no. 5, pp. 555-559, 1992.

[10] J. L. Volakis, T. Özdemir, and J. Gong, "Hybrid finite-element methodologies for antennas and scattering," IEEE Transactions on Antennas and Propagation, vol. 45, no. 3, pp. 493-507, 1997.

[11] X. Q. Sheng, J. M. Jin, J. Song, C. C. Lu, and W. C. Chew, "On the formulation of hybrid finite-element and boundaryintegral methods for 3-D scattering," IEEE Transactions on Antennas and Propagation, vol. 46, no. 3, pp. 303-311, 1998.

[12] J. M. Jin and J. L. Volakis, "A finite-element-boundary integral formulation for scattering by three-dimensional cavity-backed apertures," IEEE Transactions on Antennas and Propagation, vol. 39, no. 1, pp. 97-104, 1991.

[13] Z. Ren, "Degenerated whitney prism elements - general nodal and edge shell elements for field computation in thin structures," IEEE Transactions on Magnetics, vol. 34, no. 5, pp. 25472550, 1998.

[14] L. Lei, J. Hu, and H. Quan Hu, "Solving scattering from conducting body coated by thin-layer material by hybrid shell vector element with boundary integral method," International Journal of Antennas and Propagation, vol. 2012, Article ID 854647, 7 pages, 2012.

[15] Z. Cui, Y. Han, X. Ai, and W. Zhao, "A domain decomposition of the finite element-boundary integral method for scattering by multiple objects," Electromagnetics, vol. 31, pp. 469-482, 2011.

[16] M. M. Ilić and B. M. Notaroš, "Higher order FEM-MoM domain decomposition for 3-D electromagnetic analysis," IEEE Antennas and Wireless Propagation Letters, vol. 8, pp. 970-973, 2009.

[17] K. Umashankar, A. Taflove, and S. M. Rao, "Electromagnetic scattering by arbitrary shaped three-dimensional homogeneous lossy dielectric objects," IEEE Transactions on Antennas and Propagation, vol. 34, no. 6, pp. 758-766, 1986. 

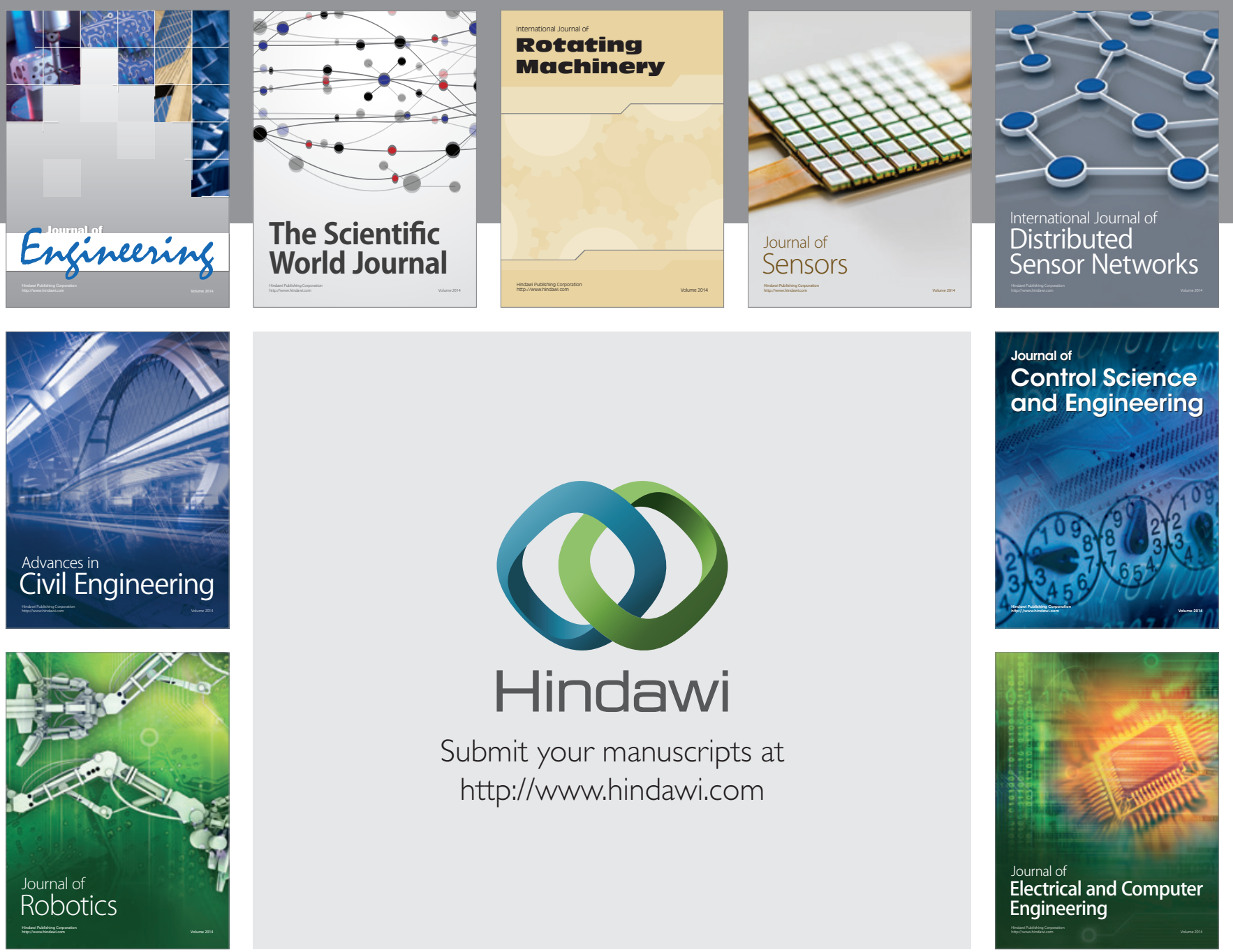

Submit your manuscripts at

http://www.hindawi.com
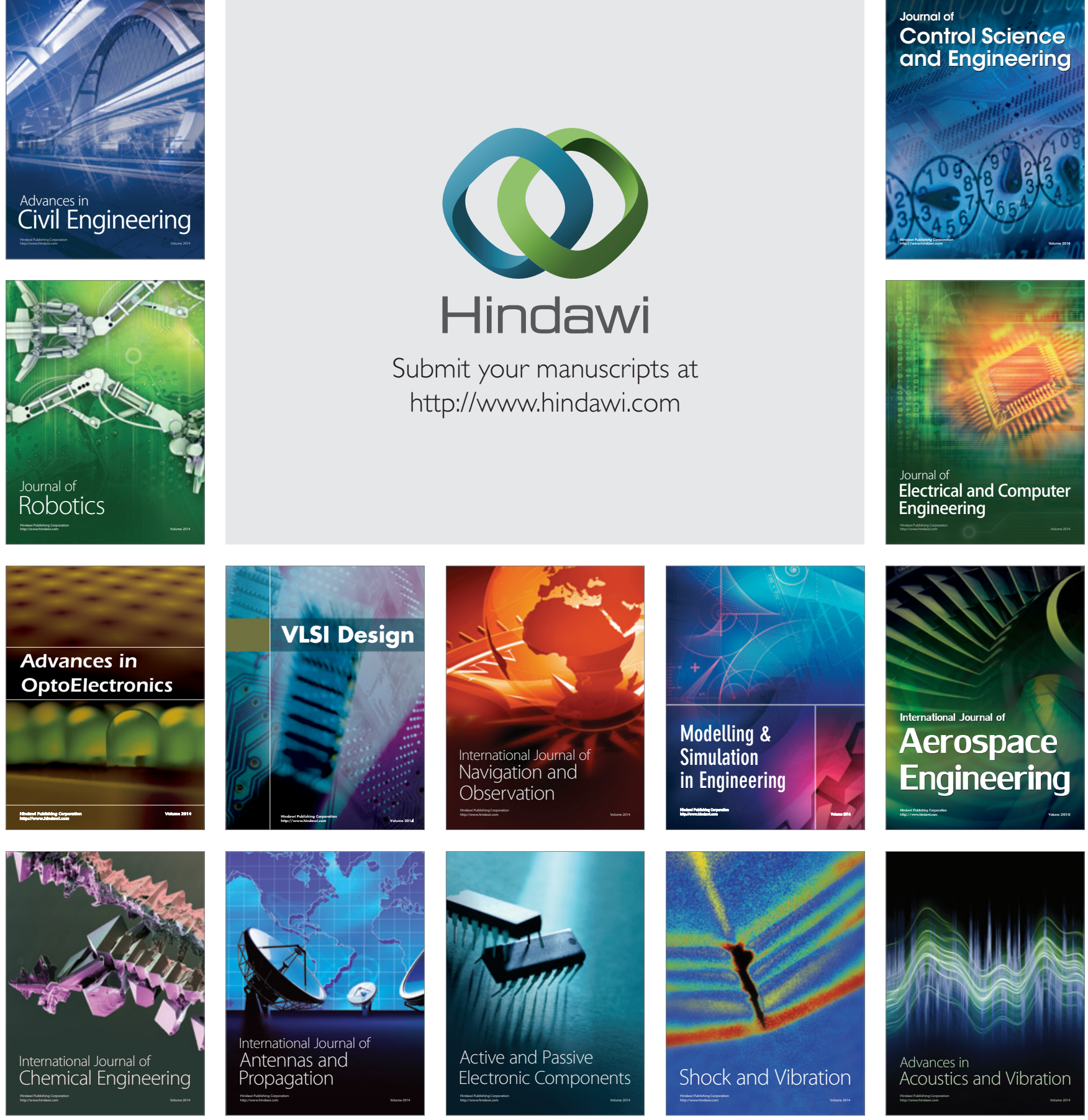